\title{
Coplanar Tricubes
}

\author{
Jean-Maurice Schramm
}

Laboratoire des Sciences de 'Image, d'Informatique et de Télédétection Université Louis Pasteur, 7, rue René Descartes, 67084 Strasbourg cedex, France e-mail :.jms@dpt-info.u-strasbg.fr

\begin{abstract}
.
Within the framework of the arithmetic discrete geometry introduced by J.P.Reveillès, 1. Debled has defined the concept of tricubes and found out that the total number of the tricubes that may appear in a naive plane is fourty.

This study concerns the coexistence of tricubes in a plane. We call complete combination of coplanar tricubes any set of tricubes, such as a naive plane exists, which contains all the tricubes of this set without any other. We present an algorithm which calculates the set of these combinations.

It appears that the number of these combinations is quite small : only 99. although a "combinatory explosion" could have been expected during their calculation. Their list is given in the appendix.
\end{abstract}

Key Words : arithmetic discrete geometry, discrete planes, Fourier's algorithm.

\section{Introduction.}

This work has been conducted in the frame of the theory of the arithmetic planes introduced by J.P.Reveillès [Rev:91], [Deb.Rev:94]. These are defined by means of two inequations. I.Debled has worked on the problem of the recognition of these planes [Deb:95]. She has introduced a local concept: the tricubes. The tricubes can be seen as little components of discrete plane, their projection on the horizontal plane being a square $3 \times 3:$ they were fourty tricubes . In another paper, J.P.Reveillès has

\footnotetext{
'This result is valid for the planes which have a certain width, called naive planes, and save on symmetries. (See §1.2). Other work [Fra, Sch, Taj: 96], using Fourier's algorithm, has led the same results.
} 
shown that each given plane could not contain more than nine different tricubes [Rev:95].

We study the compatibility between the tricubes. We call complete combination of coplanar tricubes any set of tricubes, such as a naive plane exists, which contains al! the tricubes of this set without any other. We present an algorithm which calculates the set of these combinations. In order to do this, sets of planes are associated with the different combinations of tricubes. Each one is specified by a system of linear constraints, relating to its coefficients. This approach allows to use the Fourier's algorithm.

\subsection{Outline.}

The first part of this paper defines precisely the context : we call back the definition of naive planes and tricubes (1.2). Then we define relations and functions which connect the tricubes to the planes, and we introduce the concept of complete combination of multi-coplanar tricubes which allows a simpler calculation (1.3). These definitions allow to state precisely the treated problem (1.4).

The second part contains first a study of the properties of the concepts which have been introduced (2.1). Then the properly so called calculation is approached, first the calculation of the complete combinations of multimcoplanar tricubes (2.2), then we infer the complete combinations of coplanar tricubes (2.3).

A short conclusion (3) is followed by references and results in the appendix.

\subsection{Recalls on naive planes and tricubes.}

The set of naive planes $\mathbb{P}$.

The discrete planes used in arithmetic discrete geometry (see [Rev:91], [Deb, Rev:94]), are defined as sets of points $(x, y, z)$ of $\mathbb{Z}^{3}$ satisfiying two inequations $0 \leqslant a x+b y+c z+d<\omega$, where all parameters are integers, $a, b, c$ are not all null and $\omega>0$. The discrete plane thus defined is called naive if $\omega=\sup (|a|,|b|,|c|)$. The triplet $(a, b, c)$ is called normal of the plane.

Symmetry reasons allow to limit the study to the planes defined by inequations which parameters satisfy $0 \leqslant a \leqslant b \leqslant c$, that is 1 case among 48 . Otherwise :

\section{Proposition 1.}

Two naive planes which admit the same normal can be deduced one from the other by a translation from $\mathbb{Z}^{3}$.

Proof : For the detailled proof of the stated propositions see [Sch:97].

Therefore, in this work, we consider only the discrete naive planes defined by inequations as above, but with $d=0$. All the naive discrete planes can be reduced to these by symmetries and translations. We note $\mathbb{P}$ the set of the normal corresponding to these planes. Thus, $\mathbb{P}=\left\{(a, b, c) \in \mathbb{Z}^{3} \mid 0 \leqslant a \leqslant b \leqslant c, 0<c\right\}$, but we will often interpret the elements of $P$ as the naive discrete plane they define. 


\section{Representation of some sets of planes by linear constraints systems.}

The sets of planes $P \in \mathbb{P}(\mathbb{P})^{2}$ may be infinite and so one cannot represent them in extension, that is, element by element. On the other hand, it is possible to define some of these sets by a typical property.

We consider linear constraints systems : they are expressions built from linear constraints by usual logical operators $\vee, \wedge, \rightarrow^{3}$. The linear constraints get on the coefficients $a, b, c$ which are used to specify the planes and have the form $\alpha a+\beta b$ $+\gamma c \succ \delta$, where $\alpha, \beta, \gamma, \delta \in \mathbb{Z}$ and $\succ$ is one of the comparison operators $>$, $\geqslant,<, \leqslant$. Such a constraint is called homogeneous if $\delta=0$.

When a linear constraints system is reduced to a conjunction of constraints, it is possible to use Fourier's algorithm (see [Fra,Sch,Taj:96]) to decide if the system admits a solution.

In the general case, the system can se transformed into an equivalent system in the form of a disjunction of constraints conjunctions ${ }^{4}$. Fourier's algorithm may then be used to decide the existence of solutions, but it has to be applied to each of the conjunctions of the disjunction.

This method allows particulary to test if:

- a constraint $s$ is a consequence of a system $S: S \Rightarrow s \quad$ ?

- a system $S_{1}$ implies a system $S_{2} \quad: \quad S_{1} \Rightarrow S_{2}$ ?

- two systems $S_{1}, S_{2}$ are equivalent $\quad S_{1} \Leftrightarrow S_{2} \quad$ ?

We note

- $\$$ the set of these systems,

- $\mathbb{C}$ the set of conjunctions of linear constraints (we consider that $\mathbb{C}$ is a subset of $\$$ )

- sol the function from $\mathbb{S}$ to $\mathscr{P}(\mathbb{P})$ which associates with each system of linear constraints the set of planes which satisfy this system.

The set of tricubes $\pi$.

We note $\mathbb{T}$ the set of the functions $t$ from $\{-1,0,+1\}^{2}$ to $\mathbb{Z}$ such as $t(0,0)=0 ;$ these functions are called (potential) tricubes.

\subsection{Relations between the combinations " of tricubes and the sets of planes.}

The naive discrete planes whose normal $(a, b, c)$ satisfies the conditions $0 \leqslant a \leqslant b \leqslant c$ are functional in $x, y$. For each $p \in \mathbb{P}$, tor each $x, y \in \mathbb{Z}$, we note $p^{\dagger}$ the so defined function.

\footnotetext{
${ }^{2} P(E)$ represents the set of all subsets of $E$.

${ }^{3} \vee, \wedge, \neg$ : or, and, not.

${ }^{4}$ The constraints are those of the given system or their negation.

"In this work, the word "combination" is synonymous with "set".
} 
Let $\psi$ be the function from $P \times \mathbb{Z}^{2}$ to $T$ defined by $\psi(p,(x, y))(\alpha, \beta)=p^{f}(x+\alpha, y+\beta)-p^{\prime}(x, y) \quad, \quad(\alpha, \beta \in\{-1,0,+1\})$.

This function associates with each couple formed by a naive plane $p$ and a point $(x, y)$ from the plane $\mathbb{Z}^{2}$, the tricube appearing in this plane at the point whose projection on the plane $z=0$ is the point $(x, y)$.

Let $\rho$ be the relation from $\mathbb{P}$ to $\mathbb{T}$ defined by $p(p, t) \Leftrightarrow \exists(x, y) \in \mathbb{Z}^{2}, \psi(p, x, y)=t$.

When $\rho(p, t)$ is true, one can note that $p$ contains $t$, or that $t$ appears in $p$.

Example: The following figure represents a part of the naive plane defined by the inequalities $0 \leqslant 3 x+5 y+7 z<7$ and some tricubes which appear in this plane. (The points of the plane are represented by cubes. The numbers of the tricubes are these given in the appendix A). This plane contains the tricubes $7,8,19,20,25,37$, and 40 .

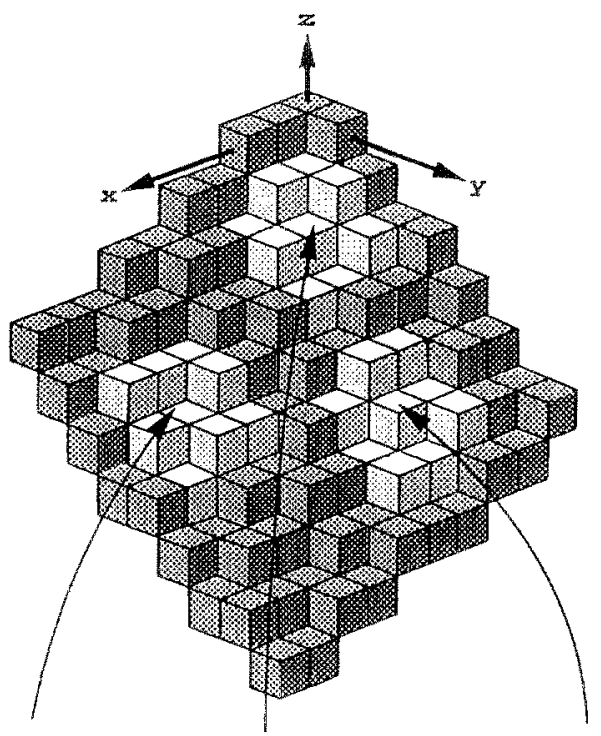

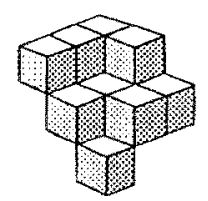

tricube 37

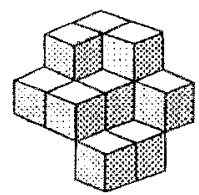

txicube 25

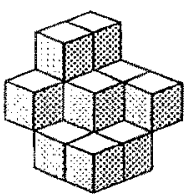

tricube 19 
As it is possible for each relation, we can define the function $\varphi$ associated with $\rho$ :

$$
\begin{aligned}
\varphi: & \mathbb{P} \rightarrow \mathbb{P}(\mathbb{T}) \\
& p \rightarrow \varphi(p)=\{t \in \mathbb{N} \mid \rho(p, t)\}=\psi\left(p, \mathbb{Z}^{2}\right) .
\end{aligned}
$$

The function $\varphi$ associates with each plane, the combination of the tricubes which appear in this plane .

We define a bijection which associates some tricubes combinations with a set of naive planes. This will help us to calculate with planes rather than directly with tricubes.

First, let $\gamma$ be the function associated with the relation $\rho^{-1}$

$$
\begin{aligned}
\gamma: \mathbb{T} & \rightarrow \mathbb{P}(\mathbb{P}) \\
& t+\gamma(t)=\{p \in \mathbb{P} \mid \rho(p, t)\} .
\end{aligned}
$$

The function $\gamma$ associates with each tricube the set of planes which contain it.

We extend $\varphi$ into the function $\phi$ :

$$
\begin{aligned}
\phi: & \mathbb{P}(\mathbb{P}) \rightarrow \mathscr{P}(\mathbb{\mathbb { T }}) \\
& P \cdots \phi(P)=\cap_{p: p}, \varphi(p) .
\end{aligned}
$$

The function $\phi$ associates with each set of planes the combination of tricubes which appears in all the planes of this set. (But these planes may contain other tricubes)

In the same way we extend $\gamma$ into the function $\Gamma$ :

$$
\begin{aligned}
\Gamma: & \mathbb{P}(\mathbb{T}) \rightarrow \mathscr{P}(\mathbb{P}) \\
& T \rightarrow \Gamma(T)=\cap_{t=1}, \gamma(t) .
\end{aligned}
$$

The function $\Gamma$ associates with each combination of tricubes the set of the planes which contain all the tricubes of the given combination (but these planes may contain other tricubes).

Eventually, we define the function $\mathrm{S}$ :

$$
\begin{aligned}
\Delta: & \mathbb{P}(\mathbb{I}) \rightarrow \mathscr{P}(\mathbb{P}) \\
& T \rightarrow \Delta(T)=\{p \in \mathbb{P} \mid \varphi(p)=T\} .
\end{aligned}
$$

The function $\Delta$ associates with each combination of tricubes the set of the planes which contain all the tricubes of the given combination and no other tricube.

We simplify the calculation by introducing the following definition :

A complete combination of multi-coplanar tricubes is a set $T$ of tricubes such as it exists a set of planes for which $T$ is the set of the tricubes appearing in all these planes.

\subsection{The problem.}

The objective of this work is to calculate the set of the complete combinations of coplanar tricubes, that is to say the set of the combinations of tricubes such as a naive 
plane exists, which contains all the tricubes of this combination, without any other. This set is $\varphi(\mathbb{P})=\Delta^{-1}(\mathscr{P}(\mathbb{P})-\{\varnothing\})$.

The calculation of $\Delta$ appeals to unions and differences (see proposition 7). This implies that the set of the planes of form $\Delta(T)$ is not always convex. For this reason, we calculate first the set $\phi(\mathbb{P}(\mathbb{P}))$ of complete combinations of multi-coplanar tricubes. Hence, we get $\Gamma(\mathscr{P}(\mathbb{T}))$ (see proposition 5 ). The elements of $\Gamma(\mathscr{P}(\mathbb{T}))$ are convex sets which can be specified by conjunctions of linear constraints.

\section{Calculation.}

\subsection{Basic properties.}

\section{Proposition 2.}

$\forall P_{1}, P_{2} \in \mathscr{P}(\mathbb{P}): \phi\left(P_{1} \cup P_{2}\right)=\phi\left(P_{1}\right) \cap \phi\left(P_{2}\right)$

$\forall T_{1}, T_{2} \in \mathbb{P}(\mathbb{T}): \Gamma\left(T_{1} \cup T_{2}\right)=\Gamma\left(T_{1}\right) \cap \Gamma\left(T_{2}\right)$.

It results that $\phi$ and $\Gamma$ are decreasing for the inclusion.

\section{Proposition 3.}

The function $\phi \circ \Gamma$ from $\mathscr{P}(\mathbb{T})$ to $\mathscr{Q}(\mathbb{T})$, and the function $\Gamma \circ \phi$ from $\mathscr{P}(\mathbb{P})$ to $\mathscr{P}(\mathbb{P})$ are increasing for the inclusion.

They can be written

It results that :

$$
\begin{aligned}
& \phi(P)=\{t \in \mathbb{J} \mid \forall p \in P, \rho(p, t)\}, \\
& \Gamma(T)=\{p \in \mathbb{P} \mid \forall t \in T, \rho(p, t)\} .
\end{aligned}
$$

\section{Proposition 4.}

For each $P$ in $\mathscr{P}(\mathbb{P})$, we have $\Gamma(\phi(P)) \supset P$ and, for each $T$ in $\mathscr{P}(\mathbb{T})$, we have $\phi(\Gamma(T)) \supset T$.

We consider the subsets of $\mathbb{P}$ which verify the property to be the set of the planes which contain (at least) a given set of tricubes. The set of the subsets of $\mathbb{P}$ which verifies such a property is the set $\Gamma(\mathscr{P}(\mathbb{U}))$.

\section{Proposition 5.}

The sets $\phi(\mathscr{P}(\mathbb{P}))$ and $\Gamma(\mathscr{P}(\mathbb{T}))$ are in one-to-one relation (by the function $\Gamma$ and $\phi$ whose restrictions are bijective).

\section{Proposition 6.}

The sets $\phi(\mathscr{P}(\mathbb{P}))$ and $\Gamma(\mathscr{P}(\mathbb{T}))$ are stable for the intersection. In fact, $\phi(\mathbb{P}(\mathbb{P}))$ is the closing of $\varphi(\mathbb{P})$ for the intersection and $\Gamma(\mathscr{P}(\mathbb{T}))$ is the one of $\gamma(\mathbb{T})$.

By an other way, the function $\Delta$ can be expressed using the function $I$ : 


\section{Proposition 7.}

$\Delta(T)=\Gamma(T)-\cup_{T^{\prime \prime} \mid T \subset T^{\prime \prime} \subset \mathbb{d}, T^{\prime} \neq T} \Gamma\left(T^{\prime}\right)=\Gamma(T) \cup_{i \in \mathbb{T}, T} \Gamma(T \cup\{t\})$.

Representation of the elements of $\Gamma(\mathscr{P}(\mathbb{T}))$ by conjunction of linear constraints. It is shown in [Fra,Sch,Taj:96] that :

Proposition 8.

$\forall t \in \mathbb{T}, \exists S \in \mathbb{C} \mid \gamma(t)=\operatorname{sol}(S)$

The sets $P$ of form $\Gamma(T)$ verify $\Gamma(T)=\cap_{t \in T} \gamma(t)$ and so can also be specified by a conjunction of linear constraints :

\section{Proposition 9.}

$\forall T \in \mathbb{P}(\mathbb{T}), \exists S \in \mathbb{C} \mid \gamma(t)=\operatorname{sol}(S)$.

The set of planes containing a given combination of tricubes may be specified by a conjunction of linear constraints.

Calculation of the set $\mathcal{T}$ of the effective tricubes.

Let $\mathcal{T}$ be the set of the values of the relation $\rho$.

$\mathcal{T}=\{t \in \mathbb{T} \mid \exists p \in \mathbb{P} \rho(p, t)\}=U_{p \in \mathbb{R}} \varphi(p)$.

The elements of $\mathcal{T}$, which are fourty, are the effective tricubes [Deb:95]. We can obtain these effective tricubes from a finite set of (potential) tricubes ${ }^{6}$ by using Fourier's algorithm to the conjunction of linear constraints which correspond to them. (See [Fra,Sch,Taj:96]). Their list is given in the appendix A.

2.2 Calculation of the set of the complete combinations of multi-coplanar tricubes $\phi(\mathscr{P}(\mathbb{P}))$.

Calculation of the set $\Gamma(\mathscr{P}(\mathbb{T}))$ by means of an automaton.

The problem for the calculation of this set is the number of elements of $\mathscr{P}(\mathbb{T})$. Of course the calculation for the $2^{40}$ subsets of $\mathcal{T}$ is not viable ! Even the subsets containing at most nine tricubes are too numerous ?

The adopted solution consists of calculating step by step, beginning with the empty set $(\Gamma(\varnothing)=\mathbb{P})$, and adding successively all the elements. So we build an automaton $\mathcal{A}$ :

This (deterministic) automaton is specified by:

- its set of states: $\Gamma(\mathscr{P}(\mathbb{T})), \quad(\subset \mathscr{P}(\mathbb{P}))$

- its alphabet : $\mathcal{T}$,

${ }^{6}$ It is easy to show that the effective tricubes verify some restrictions. For example, their image is included between -2 and +2 . It is possible to refine more (see [Yac:97]).

${ }^{7}$ J.P.Reveillès [Rev:95] has shown that it was impossible to have more than nine different tricubes in a naive plane. (He shows a more general result, where some rectangles are put in place of the $3 \times 3$ squares of the tricubes). 
- its function of transitions: $f: \Gamma(\mathscr{P}(\mathbb{T})) \times \mathcal{T} \rightarrow \Gamma(\mathbb{P}(\mathbb{T}))$,

- its initial state $: \mathbb{P}$,

$$
\Gamma(T), t \quad \rightarrow f(\Gamma(T), t)=\Gamma\left(T \cup\left\{t_{\}}\right)=\Gamma(T) \cap \gamma(t),\right.
$$

- its set of final states : $\Gamma(\mathscr{P}(\mathbb{T}))-\varnothing .^{8}$

The set of the states of the automaton is built from the initial state, by a repeated application of the transitions.

The states, represented by conjunctions of ${ }^{\prime}$ inear constraints, are stored in a list.

At the begining, the list contains only the initial state. Then, for each state figuring in the list, in order, one calculates the states to which leads the function of transition ; the so obtained states are introduced in the list, if they don't figure there yet. This process is performed until no new state appears.

This algorithm calculates $\Gamma(\mathscr{P}(\mathbb{T}))$; by a plaisant surprise, it ends quickly and gives the following result :

\section{Proposition 10.}

The set $\Gamma(\mathscr{P}(\mathbb{T}))$ has 148 elements (including $\mathbb{P}$ and $\varnothing$ ).

Calculation of the set $\phi(\mathbb{P}(\mathbb{P}))$.

Thanks to the previously described bijection (proposition 5), the automaton $\mathcal{A}$ is able to be translated into an automaton $\mathcal{A}^{\prime}$, whose states are sets of tricubes instead of sets of planes. In the usual practice, the two automatons are built simultaneously. The bijection $^{9} \phi: \Gamma(\mathbb{P}(\mathbb{T})) \rightarrow \phi(\mathscr{P}(\mathbb{P}))$ is easily obtained from the automaton $\mathcal{A}:$ $\phi(\Gamma(T))=\{t \in \mathcal{T} \mid f(\Gamma(T), t)=\Gamma(T)\}$ (set of tricubes for which the state is invariant) ${ }^{10}$

The previous result is immediately translated:

\section{Theorem 1 .}

The set $\phi(\mathbb{P}(\mathbb{P}))$ of the complete combinations of multi-coplanar tricubes has 148 elements (including $\varnothing$ et $\mathbb{T}$ ).

\footnotetext{
${ }^{8}$ The essential interest of this automaton is not the recognized language, but it is the construction of its set of states. It results that the final states have no importance. ${ }^{9} \phi$ is not a bijection. but its restriction to the indicated sets is one. See above. ${ }^{10}$ Except for the empty state $: \phi(\varnothing)=\mathbb{T}$ and not $\mathcal{T}$, but it is without importance for the following.
} 


\subsection{Calculation of the set of complete combinations of coplanar tricubes $\varphi(\mathbb{P})$} $=\Delta^{-1}(\mathbb{P}(\mathbb{P})-\varnothing)$.

The set $\varphi(\mathbb{P})$ is obviously a subset of $\phi(\mathscr{P}(\mathbb{P}))$ : one only has to restrict to the subsets of $\mathbb{P}$ with one element.

Let $T \in \phi(\mathscr{P}(\mathbb{P})): T \in \varphi(\mathbb{P}) \Leftrightarrow \Delta(T) \neq \varnothing$.

We have shown that $\Delta(T)=\Gamma(T)-U_{t=\mathbb{T}-\gamma} \Gamma(T \cup\{l\})$ and we must test if this set is empty or not.

Therefore, we represent $\Delta(T)$ by a linear constraints system :

Let $S=\pi-T$

$\Delta(T)=\Gamma(T)-U_{t \in S}(\Gamma(T) \cap \gamma(t))=\Gamma(T)-U_{i: s} \gamma(t)$

$\Delta(T)=\left\{p \mid p \in \Gamma(T) \wedge \neg\left(p \in U_{, \in S} \gamma(t)\right)\right\}=\left\{p \mid p \in \Gamma(T) \wedge\left(\wedge_{t \in S} \neg p \in \mathcal{\gamma}(t)\right)\right\}$.

Let $\left(c_{j}\right),\left(d_{l}\right),\left(e_{f}\right)$ be families of constraints such as

$p \in \Gamma(T) \Leftrightarrow \wedge_{i \in l} c_{i}, \quad p \in \gamma(t) \Leftrightarrow \wedge_{\left.i=, \mu_{i}\right)} d_{j} . \quad e_{l} \Leftrightarrow \neg d_{l}$.

Then $\Delta(T)=\left\{p \mid\left(\wedge_{1 \in I} c_{l}\right) \wedge\left(\wedge_{1 \equiv s} \neg \wedge_{t \in J(t)} d\right)\right\}$.

We transform this expression to let appear a disjunction of constraints conjunctions :

$\Delta(T)=\left\{p \mid \vee_{f \in \prod_{t \in S, J(t)}}\left(\left(\wedge_{1,1} c_{l}\right) \wedge\left(\wedge_{i, \ldots, s} e_{f t}\right)\right)\right\}$.

To know if $\Delta(T)$ is empty, it is sufficient to apply Fourier's algorithm to each of the conjunctions. From a practical view, the applied transformation (distributivity) lets appear an exponential number of conjunctions : so it is convenient to use a variant optimized for the tranformation. The algorithm provides the following result :

\section{Theorem 2 .}

The set $\varphi(\mathbb{P})$ of the complete combinations of coplanar tricubes has 99 elements. The list of these combinations is given in the appendix B.

\section{Conclusion.}

This work has given an unexpected result: there are only 99 complete combinations of coplanar tricubes (for the naive planes whose normal is in the "first" 1/48 of space). It shows once more that the Fourier's algorithm can be, even in a practical way, efficient.

Currently, the use of this result is studied in LSIIT in Strasbourg to realise a polyhedrization of discrete volumes by facetization of their sides, that is to say by decomposing them into parts of discrete planes.

To pursue this work we consider the following subjects :

- a refinement of these coplanar tricubes combinations, which could show which of the tricubes are able to be adjoining and could help to understand the structure of the discrete planes.

- the generalisation to all planes (suppression of the $1 / 48$ of space restriction). 
I whish to thank J.Françon and M.Tajine for their help and suggestions, and for the fruitful discussions we had together.

\section{Bibliography.}

To have a description and a proof of the Fourier's algorithm one can consult [Kuh:56], or the chapter 1 "Inequalities Systems" of [Sto,Wit:70]. See also the bibliography from [Fra,Sch,Taj:96]. It includes references concerning the bases of arithmetic discret geometry, Fourier's algorithm as well as some more general results of calculability.

\section{References.}

[Deb:95] I. Debled-Rennesson. Etude et reconnaissance des droites et plans discrets. Thèse de doctorat, Université Louis Pasteur, Strasbourg, 1995.

[Deb,Rev:94] 1. Debled-Rennesson, J.P. Revelllès. A new approach to digital planes. Vision Geometry III, Boston, 1994.

[Fra,Sch,Taj:96] J. Françon, J.M. Schramm, M. Tajine. Recognizing Arithmetic Straight Lines and Planes. $6^{\text {th }}$ Conference on Discrete Geometry for Computer Imagery, Lyon, 1996. Proceedings: Lecture Notes in Computer Science n'1176. Springer, 1996.

[Kuh:56] H. W. Kuhn. Solvability and consistency for linear equations and inequalities. The Americain Mathematical Monthly, vol. 63, p. 217-232, 1956.

[Rev:91] J.P. Reveillès. Géométrie discrète, calcul en nombres entiers et algorithmique. Thèse de doctorat d'état, Université Louis Pasteur, Strasbourg: 1991.

[Rev:95] J.P. Reveillès. Combinatorial pieces in digital lines and planes. Vision Geometry 4, SPIE'95, San Diego, 1995.

[Sch:97] J.M.Schramm. Tricubes coplanaires, RR97-12. LSIIT, Université Louis Pasteur, Strasbourg, 1997.

[Sto-Wit:70] J. Stoer, C. Witzgall. Convexity and Optimization in Finite Dimensions I. Die Grundlehren der mathematischen Wissenschaften in Einzeldarstellungen, Band 163, Springer, 1970.

[Yac:97] J.Yaacoub. Enveloppes convexes de réseaux et applications au traitement d'images. Thèse de doctorat, Université Louis Pasten, Strasbourg, 1997. 


\section{Appendix A : The forty effective tricubes.}

\begin{tabular}{|c|c|c|c|c|c|}
\hline & & & & \begin{tabular}{rrr|} 
& 1 & 1 \\
0 & 0 & -1 \\
0 & 0 & 0 \\
\end{tabular} & $-a-1$ \\
\hline tricube 2 & $a>=0$ & tricube 3 & $-a+b>=0$ & tricube 4 & $-a+b>0$ \\
\hline $\begin{array}{lll}-1 & -1 & -1\end{array}$ & $-2 a+b>0$ & $0-1-1$ & $2 a-b>0$ & $0-1-1$ & $-2 a-b+c>0$ \\
\hline $\begin{array}{lll}0 & 0 & 0\end{array}$ & $-2 a-b+c>0$ & $0-1 i$ & & 0 & $a>0$ \\
\hline 01 & & 01 & & 0 & $-2 b+c>0$ \\
\hline tricube 5 & $-a+b>=0$ & tricube 6 & $a>=0$ & tricube 7 & $a+b-c>0$ \\
\hline $\begin{array}{lll}0 & 0 & -1\end{array}$ & $a>0$ & $\begin{array}{lll}0 & 0\end{array}$ & $-a+b>=0$ & $-1-1-2$ & $b>0$ \\
\hline $\begin{array}{lll}0 & 0 & 0\end{array}$ & $-a-2 b+c>0$ & $\begin{array}{lll}0 & 0 & 0\end{array}$ & $-2 a-2 b+c>0$ & $0-1 i$ & $c>0$ \\
\hline $\begin{array}{lll}0 & 0 & 0\end{array}$ & & $0 \quad 01$ & & 0 & \\
\hline tricube 8 & $-2 a+c>0$ & tricube 9 & $-2 a+b>0$ & tricube 10 & $s>=0$ \\
\hline $\begin{array}{lll}-1 & -1 & -1\end{array}$ & $-a-2 b+2 c>0$ & $-1-1-1$ & $-a-b+c>0$ & $0-1-2$ & $>>0$ \\
\hline $\begin{array}{lll}0 & 0 & -11\end{array}$ & $2 a+b-c>0$ & 0001 & $2 b-c>0$ & $0-11$ & $a-2 b$ \\
\hline 01 & $2 b-c>0$ & 01 & $a>0$ & 0 & \\
\hline tricube 11 & $-a+b>=0$ & tricube 12 & $-a+b>0$ & tricube 13 & $>=0$ \\
\hline $0-1-1$ & $\begin{array}{r}2 b+2 c>0 \\
2-b>0\end{array}$ & $0-1-1$ & $-a-b+c>0$ & $\begin{array}{lll}0 & 0 & -1\end{array}$ & $>0$ \\
\hline $\begin{array}{lll}0 & 0 & -1 i\end{array}$ & $\begin{array}{l}>>0 \\
>>0\end{array}$ & 00 & $c>0$ & 01 & $2 a+2 l$ \\
\hline 10 & $2 a+b-c>0$ & 01 & $a+2 b$ & 01 & \\
\hline tricube 14 & $-a+b>=0$ & tricube 15 & $>=0$ & tricube 16 & $9>0$ \\
\hline $\begin{array}{ll}0 & 0\end{array}$ & $-a-2 b+c>0$ & $-1-1-2$ & $a+2 b-$ & $\begin{array}{lll}-1 & -1 & -1\end{array}$ & $\quad>0$ \\
\hline $\begin{array}{lll}0 & 0 & 01\end{array}$ & $a>0$ & $0-1 i$ & $-a+2 b$ & $0-1 i$ & $a+$ \\
\hline 10 & & 101 & & 1 & $-a+2 b$ \\
\hline tricube 17 & $-2 a+b>0$ & tricube 18 & $c>0$ & tricube 19 & $-2 a-b+2 c>0$ \\
\hline$-1-1-1$ & $-b+c>0$ & $0-1-2$ & $>>0$ & $0-1-1$ & $-b>0$ \\
\hline 001 & $-a+2 b-c>0$ & $0-1 i$ & $a+2 b-$ & $0-1 i$ & $c>0$ \\
\hline 01 & $a>0$ & 101 & $>0$ & 01 & $0>0$ \\
\hline tricube 20 & $-2 a+c>0$ & tricube 21 & $-a-b+c>0$ & tricube 22 & $-2 a-1$ \\
\hline $0-1-1$ & $2 a-2 b+c>0$ & $\begin{array}{lll}0 & 0 & -1\end{array}$ & $a-2 b$ & 0 & $c>0$ \\
\hline 00 & $2 b-c>0$ & $00 i$ & $a+2 b$ & 0 & $+b>0$ \\
\hline 01 & & 01 & $0>0$ & 01 & $a>0$ \\
\hline tricube 23 & $-a+b>=0$ & tricube 24 & $-a+b>=0$ & tricube 25 & $-b>0$ \\
\hline $0-1-2$ & $2 a+b-2 c>0$ & $0-1-1 ;$ & $-2 b+3 c>0$ & $\begin{array}{lll}0 & -1 & -1\end{array}$ & $2 a-b$ \\
\hline $0-1 i$ & $-b+c>0$ & $\begin{array}{lll}0 & -1 i\end{array}$ & $2 a-c>0$ & 0, & $-c>0$ \\
\hline 01 & & 01 & & 01 & $2 a-b>0$ \\
\hline tricube 26 & $\begin{array}{l}-a+b>=0 \\
2 b+2 c>0\end{array}$ & tricube 27 & $-a+b>=0$ & tricube 28 & $-a+b>=0$ \\
\hline $\begin{array}{lll}0 & 0 & -1\end{array}$ & $\begin{array}{l}-2 b+ \\
a-2 b\end{array}$ & 000 & $c>0$ & $0-1-2$ & $z>=0$ \\
\hline 0 & $2 a+b-c>0$ & $00 i$ & $2 a-b>0$ & $-1 i$ & $2 a+2 b$ \\
\hline 01 & $2 a-b>0$ & 01 & & 01 & \\
\hline tricube 29 & $-a+b>=0$ & tricube 30 & $b>0$ & tricube 31 & $>=0$ \\
\hline $0-1-1$ & $-b+c>0$ & $0-1-1$ & $c>0$ & $0-1$ & $a-2 b+c>0$ \\
\hline $0-1$, & $2 a+b-2 c>0$ & 01 & $-2 b-2 c>0$ & 0 & $2 a-c>0$ \\
\hline 01 & & 01 & $2 a-c>0$ & 2 & \\
\hline tricube 32 & $a>=0$ & tricube 33 & $a>0$ & tricube 34 & $a>0$ \\
\hline $\begin{array}{lll}-1 & -1 & -1\end{array}$ & $-b+c>=0$ & $0-1-1$ & $-b \div c>0$ & $0-1$ & $-a-b+c>0$ \\
\hline 01 & $-2 a+2 b-c>0$ & $0 i$ & $a+2 b-c>0$ & 0 & $2 b-c>0$ \\
\hline 11 & & 11 & $-2 a+b>0$ & 11 & $-2 a+b>0$ \\
\hline tricube 35 & $a>=0$ & tricube 36 & $a+b-c>0$ & tricube 37 & $a-2 b+2 c>0$ \\
\hline 0 & $-2 a-b+c>0$ & $\begin{array}{lll}0 & -1 & -1\end{array}$ & $c>0$ & $0-1$ & $2 a+b-c>0$ \\
\hline $0: 1$ & $-2 a+b>0$ & 0 & $-a+2 b-c>0$ & 0 & $2 b-c>0$ \\
\hline 11 & & 11 & $c>0$ & 1 & $-2 a+c>0$ \\
\hline tricube 38 & $-a-b+c>0$ & tricube 39 & $-b+c>-0$ & tricube 40 & $-b+c>0$ \\
\hline 00 & $a>0$ & $0-1-1$ & $a+2 b-2 c>0$ & $0-1$ & $a+b-c>0$ \\
\hline 01 & $-a+b>0$ & 01 & $-a+2 b-c>0$ & 0 & $-a+b>0$ \\
\hline 11 & & 11 & & 2 & \\
\hline
\end{tabular}




\section{Appendix B : The 99 combinations of tricubes such as it exists a naive plane which contains all the tricubes from such a combination and no other tricube.}

They are the elements from $\varphi(\mathbb{P})$.

$$
\begin{aligned}
& \{6\} \\
& \{28\} \\
& \{32\} \\
& \{2,35\} \\
& \{11,26\} \\
& \{15,39\} \\
& \{2,6,35\} \\
& \{2,32,35\} \\
& \{3,13,27\} \\
& \{8,20,37\} \\
& \{10,24,31\} \\
& \{15,28,39\} \\
& \{15,32,39\} \\
& \{1,12,21,38\} \\
& \{3,5,14,27\} \\
& \{7,19,25,40\} \\
& \{8,17,33,37\} \\
& \{10,23,29,31\} \\
& \{1,4,13,22,38\} \\
& \{1,9,20,34,38\} \\
& \{3,5,6,14,27\} \\
& \{3,5,13,14,27\} \\
& \{3,11,13,26,27\} \\
& \{7,16,20,36,40\} \\
& \{7,18,24,30,40\} \\
& \{8,11,20,26,37\} \\
& \{8,17,20,33,37\} \\
& \{8,17,32,33,37\} \\
& \{10,11,24,26,31\} \\
& \{10,23,24,29,31\} \\
& \{10,23,28,29,31\} \\
& \{1,2,12,21,35,38\} \\
& \{1,4,5,14,22,38\} \\
& \{1,9,17,33,34,38\} \\
& \{1,11,12,21,26,38\} \\
& \{7,11,19,25,26,40\} \\
& \{7,15,19,25,39,40\} \\
& \{7,16,17,33,36,40\} \\
& \{7,18,23,29,30,40\} \\
& \{1,2,4,13,22,35,38\} \\
& \{1,2,9,20,34,35,38\} \\
& \{1,3,4,13,22,27,38\} \\
& \{1,3,12,13,21,27,38\} \\
& \{1,4,5,6,14,22,38\} \\
& \{1,4,5,13,14,22,38\} \\
& \{1,4,12,13,21,22,38\} \\
& \{1,8,9,20,34,37,38\} \\
& \{1,8,12,20,21,37,38\} \\
& \{1,9,12,20,21,34,38\} \\
& \{1,9,17,20,33,34,38\} \\
& \{
\end{aligned}
$$

